\title{
ON RING EXTENSIONS OF FSG RINGS
}

\author{
LE VAN THUYET
}

\begin{abstract}
A ring $R$ is called right FSG if every finitely generated right $R$-subgenerator is a generator. In this note we consider the question of when a ring extension of a given right FSG ring is right FSG and the converse. As a consequence we obtain some results about right FSG group rings.
\end{abstract}

\section{INTRODUCTION}

In this note all rings are associative with identities and all modules are unitary. For a ring $R$, the category of all right (left) $R$-modules is denoted by $\operatorname{Mod}-R$ ( $R$-Mod). Let $M_{R}$ be a right $R$-module. A module $N$ is called $M$-generated (or $M$ generates $N$ ) if there exists a set $A$ and an epimorphism $M^{(A)} \rightarrow N$, where $M^{(A)}$ is the direct sum of $|A|$ copies of $M(|A|$ denotes the cardinality of the set $A)$. When $A$ is finite, we say that $N$ is $M$-finitely generated. $N$ is called $M$-cogenerated (or $M$ cogenerates $N$ ) if there exist a set $A$ and a monomorphism $N \rightarrow M^{A}$ is the direct product of $|A|$ copies of $M$. When $A$ is finite, we say that $N$ is $M$-finitely cogenerated. For a module $M_{R}$, we denote by $\sigma[M]$ the full subcategory of Mod- $R$ whose objects are submodules of $M$-generated modules (see [11]).

For a right $R$-module $M$, the trace ideal of $M$ in $R$ is denoted by trace $(M)$. By definition, $\operatorname{trace}(M)=\sum\left\{\operatorname{im} \varphi: \varphi \in \operatorname{Hom}_{R}\left(M, R_{R}\right)\right\}$ (see [11, p.154]).

A module $M_{R}$ is called faithful if $\{a \in R: M a=0\}=0$. Then $M$ is faithful if and only if $M$ cogenerates every projective right $R$-module. Dually, a module $M_{R}$ is called cofaithful if $M$ generates every injective right $R$-module (see [1, p.217]). It follows that $M$ is cofaithful if and only if there exists a finite subset $\left\{m_{1}, \ldots, m_{n}\right\}$ of elements of $M$ such that $\left\{x \in R: m_{1} x=\ldots=m_{n} x=0\right\}=0$. By Lemma 1 below we see that $M_{R}$ is cofaithful if and only if $\sigma\left[M_{R}\right]=\operatorname{Mod}-R$. A module $M_{R}$ with this property is called a subgenerator of Mod- $R$ (see Wisbauer [11, p.118]). Therefore, instead of cofaithful right $R$-modules we shall use the terminology "right $R$-subgenerators".

Received 8 June 1993

I wish to thank Professors Dinh van Huynh and John Clark for many helpful and stimulating discussions during the preparaion of this paper. I am also very much indebted to Professor Nguyen H.V. Hung for calling my attention to the study of ring extensions and FSG group rings. I would also like to thank the Referee for suggestions that improved the presentation.

Copyright Clearance Centre, Inc. Serial-fee code: 0004-9729/94 \$A2.00+0.00. 
Let $R$ be a ring and $G$ a group. Then by $R[G]$ we denote the group ring of $R$ over $G$.

A ring $R$ is called right FPF if every finitely generated faithful right $R$-module is a generator. For details about FPF rings we refer to Faith and Page [6] and Faith and Pillay [7]. We introduce the family of right FSG rings as a generalisation of the class of right self-injective rings and the class of right FPF rings: A ring $R$ is called right FSG if every finitely generated right $R$-subgenerator is a generator. Basic results about FSG rings were obtained in $[\mathbf{8}]$.

Let $A$ and $B$ be rings. If $A$ is a subring of $B$ with common identity, then we say that $B$ is a ring extension of $A$. In this paper, we shall consider conditions under which a ring extension $B$ of a given right FSG ring $A$ becomes right FSG and conversely.

\section{RESULTS}

First we list some known results used in this section.

Lemma 1. Let $M_{R} \in \operatorname{Mod}-R$. Then the following conditions are equivalent:

(i) $M_{R}$ is a cofaithful module.

(ii) There exists a finite set $\left\{m_{1}, \ldots, m_{n}\right\}$ of elements of $M$ such that $\left\{x \in R: m_{1} x=\ldots=m_{n} x=0\right\}=0$,

(iii) There exists a positive integer $n$ such that $R_{R}$ can be embedded into $M^{n}$.

(iv) $M$ generates every injective right $R$-module.

(v) $\sigma[M]=\operatorname{Mod}-R$.

(vi) Cyclic submodules of $M^{(\mathrm{N})}$ form a set of generators in Mod-R.

Proof: See [1, Exercise 18.25,p.217], [2, Proposition 4.5.4] and [11, 15.3].

Recall that a ring $R$ is strongly right bounded if every nonzero right ideal contains a nonzero ideal. A commutative ring is strongly right (and left) bounded.

LEMMA 2. If $R$ is a strongly right bounded right FSG ring then $R$ is right FPF. In particular any commutative FSG ring is FPF.

Proof: Let $R$ be a strongly right bounded right FSG ring and let $M$ be a finitely generated faithful right $R$-module, say $M=x_{1} R+\cdots+x_{n} R$. Set $A=$ $r\left(\left\{x_{1}, \ldots, x_{n}\right\}\right)$. If $A \neq 0$, there is a nonzero ideal $B$ of $R$ such that $B \subseteq A$. Then $M B=\left(x_{1} R+\cdots+x_{n} R\right) B=x_{1} B+\cdots+x_{n} B=0$, a contradiction. Hence $A=0$ and so $M$ is a subgenerator and then a generator of Mod-R. This means that $R$ is right FPF.

The following result provides sufficient conditions for a ring extension of a right FSG to be right FSG. 
ThEOREM 3. Let $B$ be a ring extension of $A$ such that:

(a) $B$ is finitely generated as a right $A$-module,

(b) $B$ generates $B \otimes_{A} B$ as $B$-bimodules.

If $A$ is right FSG so is $B$.

Proof: Let $X$ be a finitely generated right $B$-subgenerator. It is easy to see that $X$ is a finitely generated right $A$-subgenerator. By assumption, $X_{A}$ is a generator. We shall prove that $X$ is a generator in Mod- $R$.

From (b), we have an exact sequence as $B$-bimodules:

$$
\underset{I}{\oplus} B \longrightarrow B \otimes_{A} B \longrightarrow 0
$$

for some index set $I$.

By tensoring (1) with $X_{B}$, we have the following commutative diagram in Mod- $B$ with exact rows:

$$
\begin{aligned}
& X \otimes_{B}\left(\Theta_{I} B\right) \longrightarrow X \otimes_{B}\left(B \otimes_{A} E\right) \longrightarrow 0 \\
& \begin{array}{c}
\text { l| } \\
\oplus_{I}\left(X \otimes_{B} B\right) \longrightarrow\left(X \otimes_{B} B\right) \otimes_{A} B \longrightarrow 0
\end{array} \\
& \text { 21 } 21 \\
& \oplus_{I} X \quad \longrightarrow \quad X \otimes_{A} B \longrightarrow 0
\end{aligned}
$$

It follows from this that $X$ generates $X \otimes_{A} B$.

Since $X$ is a generator in $\operatorname{Mod}-A$, we obtain an exact sequence in $\operatorname{Mod}-A$ :

$$
X^{n} \longrightarrow A \longrightarrow 0
$$

for some positive integer $n$.

Tensoring (2) with ${ }_{A} B$, we have the following commutative diagram with exact rows in Mod- $B$ :

$$
\begin{array}{cc}
X^{-i} \otimes_{A} B & \longrightarrow A \omega_{A} B \longrightarrow 0 \\
2 \mid & \geqslant \mid \\
\left(X \otimes_{A} B\right)^{t} \longrightarrow & \longrightarrow B \longrightarrow 0 .
\end{array}
$$

Hence $X \otimes_{A} B$ is a generator in Mod- $B$. This proves that $X$ is a generator in Mod- $B$. Thus $B$ is a right FSG ring.

Similar to [5], we have a result about group rings. 
Propos ITION 4. Let $R$ be a ring and $G$ a finite group. If $R$ is right FSG then $R[G]$ is right FSG.

Proof: Let $G=\left\{g_{1}, \ldots, g_{n}\right\}$ and let $M$ be a finitely generated subgenerator in Mod- $R[G]$. It is easy to see that $M$ is a finitely generated right $R$-module. Moreover, $M$ is a subgenerator in Mod- $R$. Indeed, let $\left\{m_{1}, \ldots, m_{t}\right\} \subset M$ such that $r_{R[G]}\left(\left\{m_{1}, \ldots, m_{t}\right\}\right)=0$. Then if $c \in r_{R}\left(\left\{m_{1}, \ldots, m_{t}\right\}\right)$, that is, $m_{1} c=\ldots=m_{t} c=$ 0 , it follows that $c=0$. By assumption, $M$ is a generator in Mod- $R$.

We have:

$$
\operatorname{trace}_{R[G]} M=\bigoplus_{i=1}^{n} \operatorname{trace}_{g_{i} R} M=\bigoplus_{i=1}^{n} g_{i} R=R[G],
$$

proving that $M$ is a generator in $\operatorname{Mod}-R[G]$.

Now we present an example of a right and left FSG ring whose centre is not FSG. Another example of the subring of elements fixed by a finite group of automorphisms of an FSG ring which need not be FSG is presented here.

EXAmPLE 5: (Clark $[3,4]$ ). Let $K$ be a field of two elements and $G$ the quaternion group of order eight, that is

$$
G=\left\langle a, b: a^{4}=b^{4}=1, a^{2}=b^{2}, b^{-1} a b=a^{-1}\right\rangle .
$$

Then we have the group ring $R=K[G]$. Since $G$ is finite, $R$ is self-injective by a result of Renault $[9,10]$. Thus $R$ is FSG by $[2$, Proposition 4.5.6].

By [3], $C$, the centre of $R$ is not FPF. By Lemma 2, $C$ is not FSG.

Also, by [4], $G$ has automorphism group

$$
S_{4}=\left\langle g, h: g^{4}=h^{2}=(g h)^{3}=1\right\rangle
$$

where $g(a)=a, g(b)=a b, h(a)=b$, and $h(b)=a$. Now let $F$ denote the group of automorphisms of $R$ obtained by extending linearly to $R$ the action of $S_{4}$ on $G$. Then it is easy to check that:

$$
R^{F}:=\{r \in R: \forall g \in F(g(r)=r)\}=\left\{0,1, a^{2}, 1+a^{2}, w, 1+w, a^{2}+w, 1+a^{2}+w\right\}
$$

where $w=a+a^{3}+b+a b+a^{2} b+a^{3} b$. Moreover, $R^{F}$ is commutative and not FPF. By Lemma 2, $R^{F}$ is not FSG.

However, in the following results we shall consider the FSG ring extension of a ring $A$ with some additional condition for which $A$ becomes a FSG ring.

Recall a module $M_{R}$ is called a torsionless module if for each non-zero element $x$ in $M$ there exists an $R$-homomorphism $f$ from $M$ to $R_{R}$ such that $f(x) \neq 0$. For example, every projective module is torsionless.

Let $\mathrm{R}$ be a ring. For a subset $X$ of $R$, we denote by $V_{R}(X)$ the subring of $R$ consisting of all $r$ in $R$ such that $r x=x r$ for all $x$ in $X$. 
Theorem 6. Let $B$ be a ring extension of $A$ such that:

(a) $B$ is torsionless as a left $A$-module,

(b) $B$ is a generator in $\operatorname{Mod}-A$,

(c) $V_{B}(A)$ generates $B$ as a $A$-module.

Then if $B$ is a right FSG ring, so is $A$.

Proof: Let $B$ be a ring extension of $A$ such that $B$ and $A$ satisfy (a), (b) and (c). Assume that $B$ is right FSG and $Y$ is a finitely generated subgenerator in Mod- $A$. Set $X=Y \otimes_{A} B$. Then $X$ is a finitely generated right $B$-module. Moreover, $X$ is a subgenerator in Mod- $B$. Indeed, since $Y_{A}$ is a subgenerator, there exists $\left\{y_{1}, y_{2}, \cdots, y_{n}\right\} \subset Y$ such that $r_{A}\left(\left\{y_{1}, y_{2}, \cdots, y_{n}\right\}\right)=0$. Assume that

$$
\left(y_{1} \otimes 1\right) b=\ldots=\left(y_{n} \otimes 1\right) b=0
$$

for some $b$ in $B$. Let $f$ be any homomorphism from $B$ to $A$ in $A$-Mod. Then $y_{1} f(b)=\ldots=y_{n} f(b)=0$. Hence $f(b)=0$. By $(a), b=0$. This proves that

$$
r_{B}\left(\left\{y_{1} \otimes 1, \cdots, y_{n} \otimes 1\right\}\right)=0
$$

that is, $X$ is a subgenerator in Mod- $B$. By assumption, $X$ is a generator in Mod- $B$. Hence $X$ is a generator in $\operatorname{Mod}-A$ by (b).

$\mathrm{By}(\mathrm{c})$ we have an exact sequence of $A$-bimodules:

$$
\underset{I}{\oplus} A \longrightarrow B \longrightarrow 0
$$

for some index set $I$.

Tensoring (3) with $Y_{A}$ gives the following commutative diagram with exact rows in Mod- $A$.

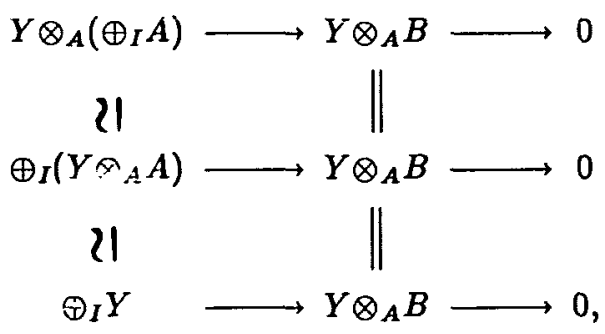

that is, $Y$ generates $X=Y \oplus_{A} B$ in Mod- $A$. It follows that $Y$ is a generator in Mod- $A$. Hence $A$ is right FSG. 
Corollary 7. Let $B$ be a ring extension of $A$ such that $A$ finitely generates $B$ as an $A$-bimodule. If $B$ is right (respectively left) FSG, then $A$ is right (respectively left) FSG.

Proof: Since $A$ finitely generates $B$ as $A$-bimodules, there exist $v_{1}, \cdots, v_{n}$ in $V_{B}(A)$ and $f_{1}, \cdots, f_{n}$ in $\operatorname{Hom}\left({ }_{A} B_{A},{ }_{A} A_{A}\right)$ such that

$$
\sum_{i=1}^{n} f_{i}(b) v_{i}=b
$$

for all $b$ in $B$. Let $C$ be the centre of $A$. Let us define mappings:

$$
\begin{gathered}
g: V_{B}(A) \otimes C A \longrightarrow B \\
v \otimes a \longmapsto v a \\
h: B \longrightarrow V_{B}(A) \otimes_{C} A \\
b \longmapsto \sum_{i=1}^{n} v_{i} \otimes f_{i}(b) .
\end{gathered}
$$

Then $g$ and $h$ are mutually inverse mappings. Since $f_{i}\left(V_{B}(A)\right) \subset C, V_{B}(A)$ is a finitely generated projective $C$-module, hence it is a generator. It follows that $B$ is a generator as a right $A$-module. By the same argument as in proving Theorem 6 , we obtain that if $B$ is right FSG then so is $A$. Similarly, if $B$ is left FSG then so is $A$. D

COROLLARY 8. Let $R$ be a ring and $G$ a group. If the group ring $R[G]$ is right (respectively left) FSG, then $R$ is right (respectively left) FSG. Moreover, if $G$ is finite, then $R$ is right (respectively left) FSG if and only if $R[G]$ is right (respectively left) FSG.

Proof: By Theorem 6 and Proposition 4.

Concerning this Corollary 8 we note that Connell [5] proved that, if $G$ is a finite group then the group ring $R[G]$ is right self-injective if and only if $R$ is right selfinjective.

\section{REFERENCES}

[1] F.W. Anderson and K.R. Fuller, Rings and categories of modules (Springer-Verlag, Berlin, Heidelberg, New York, 1974).

[2] J.A. Beachy, 'Generating and cogenerating structures', Trans. Amer. Math. Soc. 158 (1971), 75-92.

[3] J. Clark, 'The centre of an FPF ring need not be FPF', Bull. Austral. Math. Soc. 37 (1988), 235-236. 
[4] J. Clark, 'A note on the fixed subring of an FPF ring', Bull. Austral. Math. Soc. 40 (1989), 109-111.

[5] I. Connell, 'On the group ring', Canad. J. Math. 15 (1963), 650-685.

[6] C. Faith and S. Page, FPF ring theory: Faithful modules and generators of Mod- $R$, London Math. Soc. Lecture Notes Series 88 (Cambridge Univ. Press, Cambridge, 1984).

[7] C. Faith and P. Pillay, Classification of commutative FPF rings, Notas de Mathematica 4 (Universidad de Murcia, 1990).

[8] Le Van Thuyet, 'On rings whose finitely generated cofaithful modules are generators', Algebra Ber. München 70 (1993), 1-38.

[9] G. Renault, 'Sur les anneaux de groups', C.R. Acad. Sci. Paris Sér A-B 273 (1971), 84-87.

[10] G. Renault, 'Sur les anneaux de groupes', in Rings, modules and radicals 6, Proc. Colloq., Keszthely 1971, pp.371-396 (Colloq. Math. Soc. János Bolyai, North-Holland Amsterdam, 1973).

[11] R. Wisbauer, Grundlagen der modul-und ringtheorie (R. Fischer Verlag, München, 1988).

Department of Mathematics

Hue University of Education

32 Le Loi St

Hue

Vietnam 